Supporting Information

\title{
Sequential Self-Assembly of Organic Heterostructured Architectures Composed of Low-Dimensional Microcrystals
}

Mao-Lin Pan, Cheng Wang, Ming-Peng Zhuo*, Zhi-Zhou Li, Yi-Yuan, Yi-Chen Tao, and Xue-Dong Wang*

Jiangsu Key Laboratory for Carbon-Based Functional Materials \& Devices, Institute of Functional Nano \& Soft Materials (FUNSOM), Soochow University, Suzhou, Jiangsu 215123, R. R. China.

*E-mail: wangxuedong@suda.edu.cn (X.-D. Wang); mpzhuo@suda.edu.cn (M.-P. Zhuo). 


\section{Experimental details}

\section{Materials}

The compound of 2,2'-((1E,1'E')-1,4-plenylenebis(ethene-2,1diyl))- dibenzonitride (o-BCB) and 2,5,8,11-Tetra-tert-butylperylene (TBPe) were purchased from Sigma-Aldrich and were used without further treatment. The compound 1,4-dimethoxy-2,5-di (E)-bis(2-methylstyryl) benzene $(m$-BCB) was synthesized by ourselves. The solvent of dichloromethane (DCM, HPLC grade), tetrahydrofuran (THF, HPLC grade), methanol (HPLC grade ) and ethanol (HPLC grade ) were all purchased form Beijing Chemical Agent Ltd., China. All the solvents were used without any further treatment.

\section{Preparations of organic microcrystals}

In a typical preparation process, $9.97 \mathrm{mg}(3 \mathrm{mM}) o$-BCB powder was well dissolved in $10 \mathrm{~mL}$ tetrahydrofuran (THF), then the solution was directly dropped onto a quartz substrate by a pipettor at room temperature in the air environment, after which the singlecrystalline microbelts were obtained after complete evaporation. Similarly, $4.8 \mathrm{mg}(10 \mathrm{mM}) \mathrm{TBPe}$ powder was dissolved in $1 \mathrm{~mL}$ mixed solvent of DCM and methanol with a volume ratio of 1:1 and $7.89 \mathrm{mg}(2 \mathrm{mM}) m$-BCB powder was dissolved in $10 \mathrm{~mL}$ mixed solvent with $V_{\mathrm{DCM}}: V_{\text {ethanol }}=1: 2$. Both solutions were then dropped onto quartz substrates at room temperature in the air environment to form singlecrystalline microplates and microwires by evaporation.

\section{Preparations of organic heterostructures.}


For $o$-BCB/TBPe heterostructures, $1.2 \mathrm{mg}(3.5 \mathrm{mM}) o$-BCB and $2.4 \mathrm{mg}(5 \mathrm{mM}) \mathrm{TBPe}$ powder were dissolved in a mixed solvent comprising $500 \mu \mathrm{L}$ DCM and $500 \mu \mathrm{L}$ methanol, then the solution was dropped onto a quartz substrate at room temperature in the air environment before evaporation. In the preparation of $m$-BCB/TBPe heterostructures, phase separation tends to happen. The optimal way is to dissolve $0.8 \mathrm{mg}(2 \mathrm{mM}) m$-BCB and 2.4 $\mathrm{mg}(5 \mathrm{mM})$ TBPe powder in $1 \mathrm{~mL}$ mixed solvent of DCM and ethanol with a volume ratio of $1: 2$, then the solution was dropped on a quartz substrate within a short period of time after its preparation.

\section{Characterizations of the organic microcrystals}

The topography, morphology and size of microstructures were observed by scanning electron microscopy (SEM, Carl Zeiss, Supra 55, Germany) with an accelerating voltage of $10 \mathrm{kV}$ on an indium tin oxide (ITO) coated glass and transmission electron microscopy (TEM, FEI company, Tecnai G2 F20, United States) with an accelerating voltage of $20 \mathrm{kV}$ on a carbon-coated copper grid. The selected area electron diffraction (SAED) test was conducted in the TEM with an accelerating voltage of $120 \mathrm{KV}$. The X-ray diffraction (XRD) patterns were measured by a D/max $2400 \mathrm{X}$-ray diffractometer with $\mathrm{Cu} \mathrm{K} \alpha$ radiation $(\lambda=$ $1.54050 \AA$ ) operated in the $2 \theta$ range from $5^{\circ}$ to $40^{\circ}$ from samples on the quartz substrate.

Fluorescence images were recorded using a fluorescence optical microscope (Leica, DM4000M, Germany) with a spot-enhanced charge couple device (Diagnostic Instrument, Inc.). The steady-state fluorescence spectra of the samples were measured on a HITACHI F-4600 fluorescence spectrophotometer. Micro-area photoluminescence ( $\mu$-PL) spectra were 
collected on a homemade optical microscopy and the equipment were set up as shown in the Scheme S1. A $375 \mathrm{~nm}$ laser (Continuum, SURELITE II-10) was focused on a spot with diameter of $2 \mu \mathrm{m}$ to excite the individual microstructure. The emission light passed through the dichroic mirror and was collected by a thermal-electrically cooled CCD (Princeton Instruments, PIX-256E) and analyzed by a spectrograph (Princeton, ARC-SP-2356). The size and position of PL spectra collection region can be adjusted by a grating. In addition, the time-resolved fluorescence decay of these samples was also measured with a HORIBA JOBTN YVON FLUOROMAX-4 spectrofluorimeter.

\section{Theoretical calculations}

The $o$-BCB crystal, $m$-BCB crystal and TBPe crystal morphologies were calculated by the Materials Studio software, based on the attachment energy $\left(E_{\text {att }}\right)$ theory. Typically, the molecular arrangements of the crystals were firstly obtained based on the experimental crystal structure (a CIF file). After importing the CIF file, the attachment energies will be calculated by $\quad$ 'Build $\rightarrow$ Bonds $\rightarrow$ Modules $\rightarrow$ Forcite $\rightarrow \quad$ Calculation $\rightarrow$ Geometry optimization $\rightarrow$ Run' and the geometric morphology was performed using the Forcite and Morphology $\quad$ modules $\quad$ (Modules $\rightarrow$ Morphology $\rightarrow \quad$ Calculation $\rightarrow$ Growth morphology $\rightarrow$ Compass $\rightarrow$ Run). Then the predicted morphologies of the crystal could be achieved. 


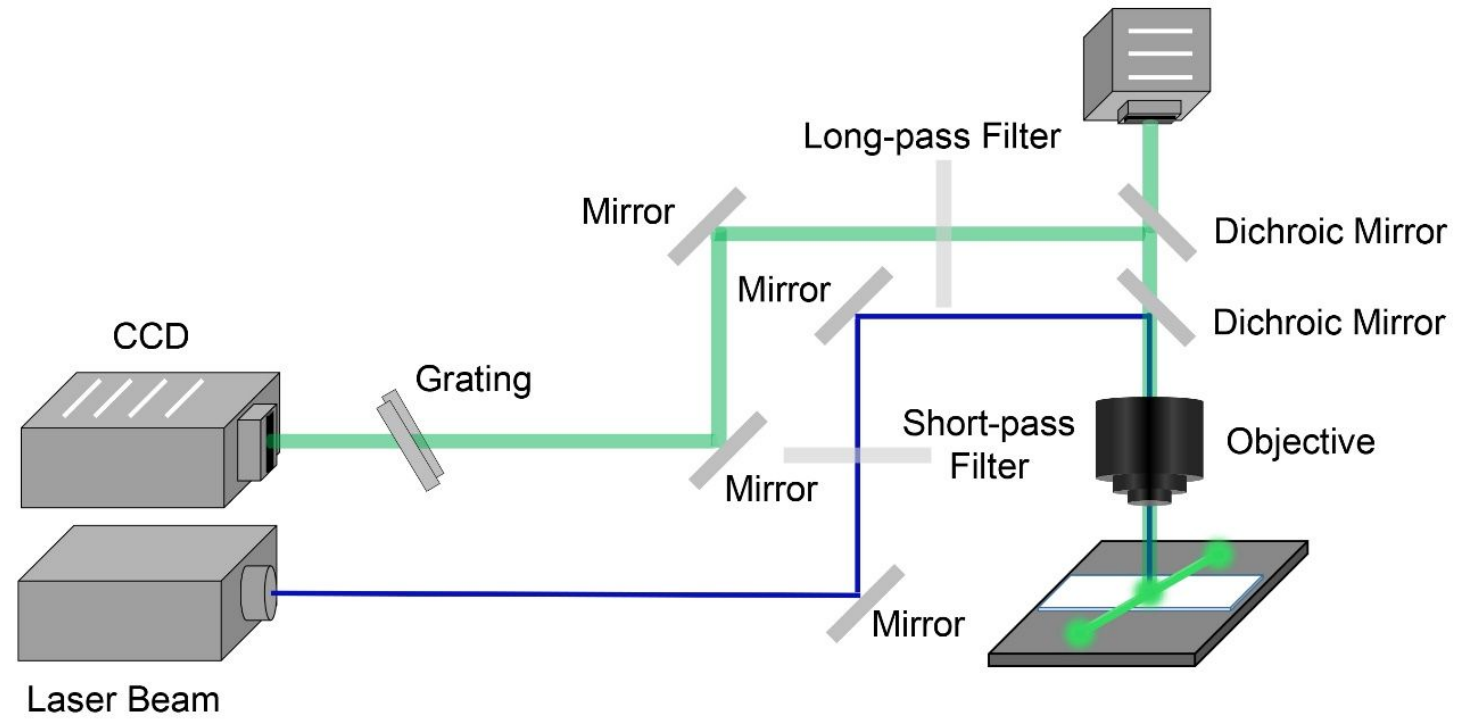

Scheme S1. Schematic illustration of the laser micro-area-spectral analyzer system.

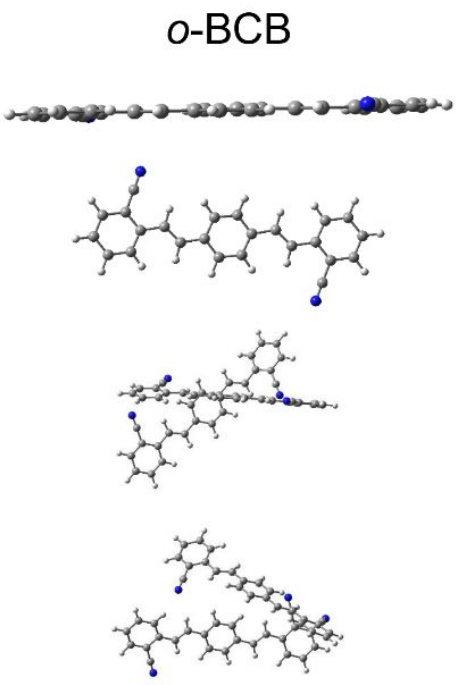

$-25.56 \mathrm{Kcal} \mathrm{mol}^{-1}$
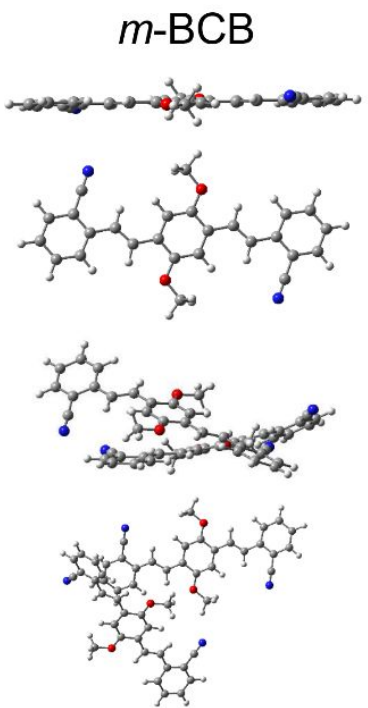

$-15.46 \mathrm{Kcal} \mathrm{mol}^{-1}$
TPBe
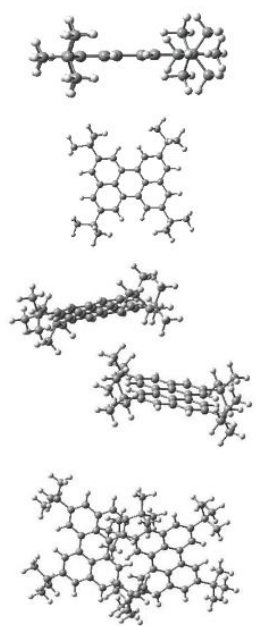

$-5.06 \mathrm{Kcal} \mathrm{mol}^{-1}$

Figure S1. The top and side views of molecular configurations of $o$-BCB, $m$-BCB and TBPe molecules. The DFT calculations for the bond energy intensity: $E_{o-\mathrm{BCB}}=-25.56 \mathrm{kcal} \mathrm{mol}^{-1}$, $E_{m-\mathrm{BCB}}=-15.46 \mathrm{kcal} \mathrm{mol}^{-1}, E_{\mathrm{TPBe}}=-5.06 \mathrm{kcal} \mathrm{mol}^{-1}$. 


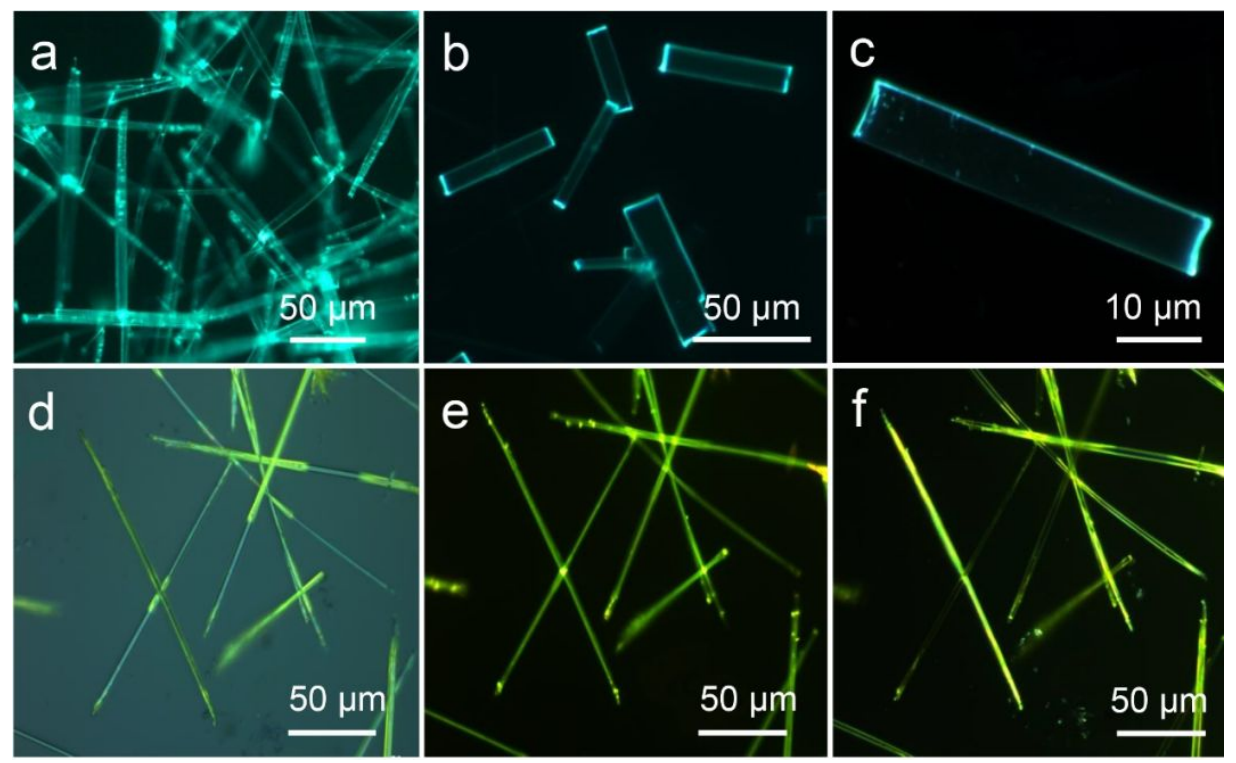

Figure S2. (a-c) The fluorescence microscope images of the $o-\mathrm{BCB}$ microbelts with an increasing magnification. The (d) bright field, (e) fluorescence and (f) dark field microscope images of the same $m$-BCB microwire. The scale bar of (c) is $10 \mu \mathrm{m}$. The other scale bars are all $50 \mu \mathrm{m}$.

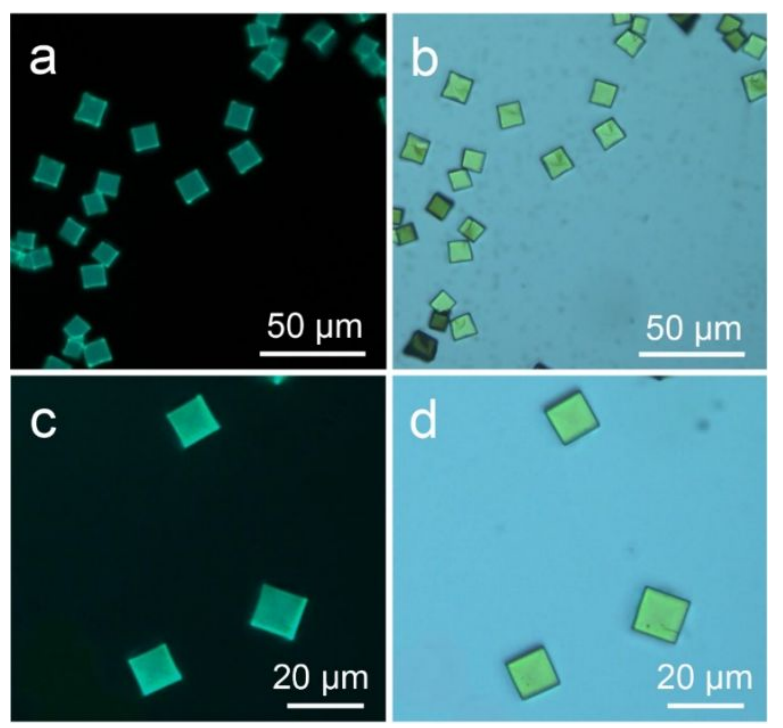

Figure S3. (a, c) The bright field microscope images of the TBPe microplates. (b, d) The fluorescence microscope images of the TBPe microplates. The scale bar of (a) and (b) are 50 $\mu \mathrm{m}$, as well as the scale bar of (c) and (d) are $20 \mu \mathrm{m}$. 


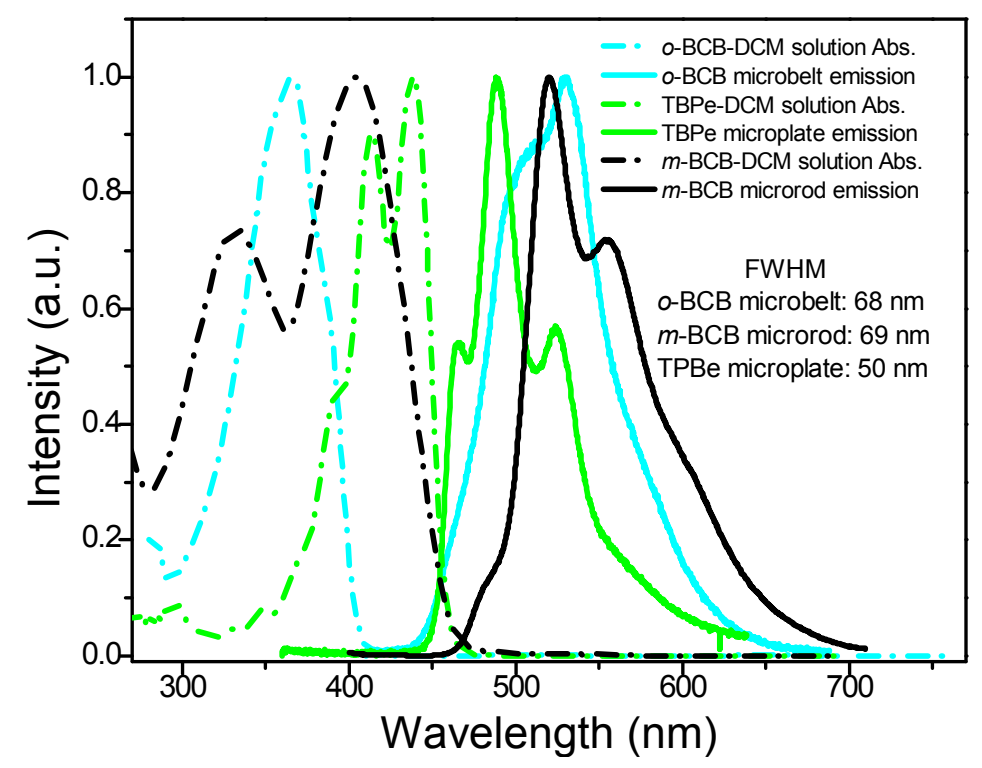

Figure S4. The fluorescence (solid line) and absorption (dashed line) spectrum of the $o$-BCB (green-blue line), $m$-BCB (black line) and TPBe (green line).
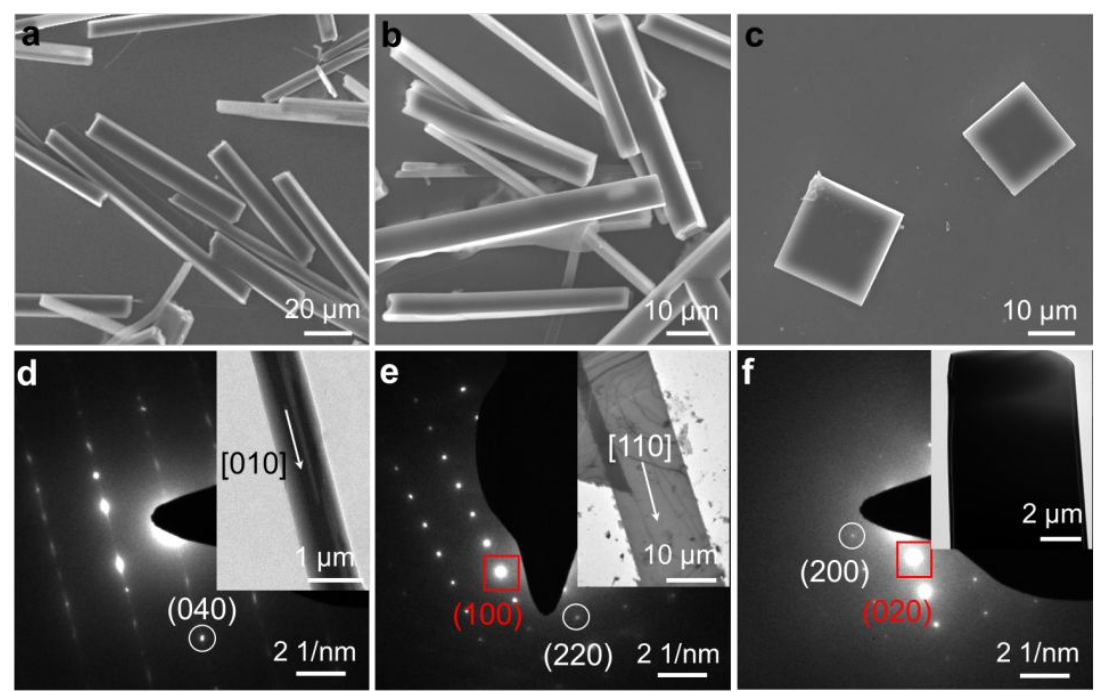

Figure S5. (a-c) SEM images and (d-f) the corresponding SAED patterns of $o$-BCB microbelts, $m$-BCB microwires and TBPe microplates, respectively. The insets in (d-f) are the TEM images of the single crystals where the SAED patterns was collected. The scale bar of a is $20 \mu \mathrm{m}$, as well as the scale bars of (b) and (c) are $10 \mu \mathrm{m}$. The scale bar of (d-f) are 2 $1 / \mathrm{nm}$. 

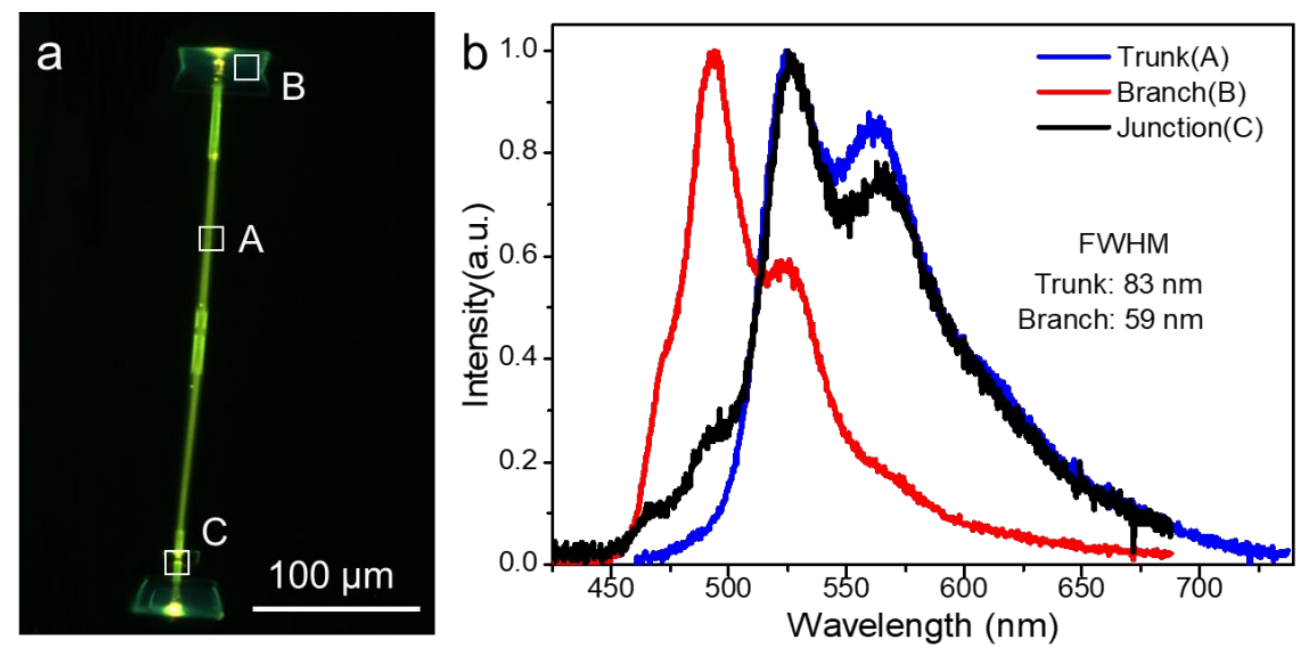

Figure S6. (a) The fluorescence microscope image of a typical $m$-BCB/TBPe BHS. The scale bar is $100 \mu \mathrm{m}$. (b) The micro-area spectra of the corresponding marked zones in (a).
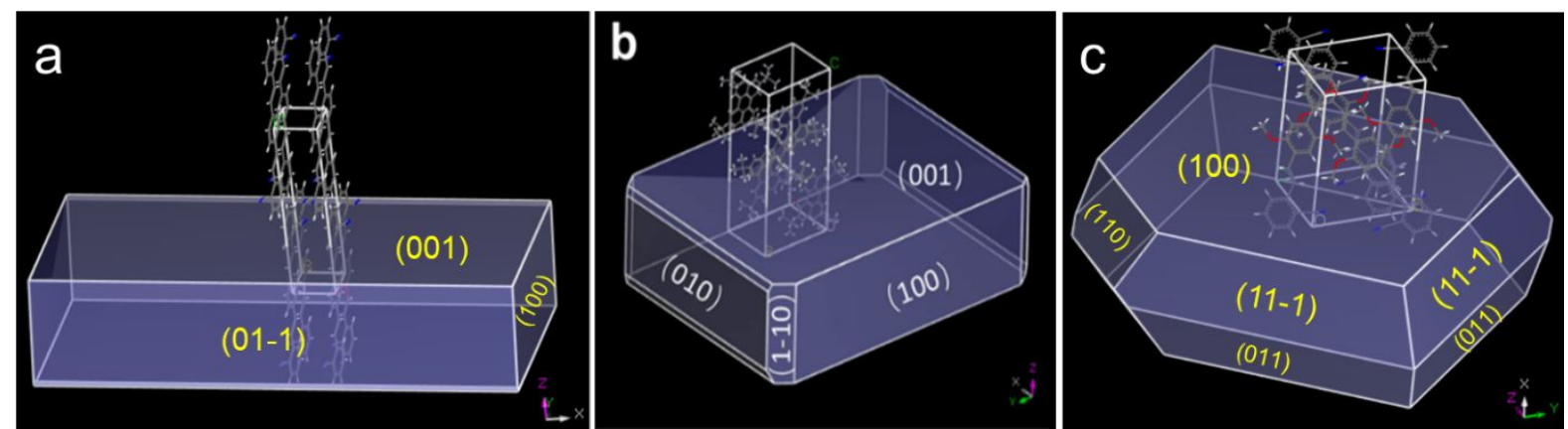

Figure S7. The simulated growth morphology of (a) $o$-BCB molecules, (b) TBPe molecules and (c) $m$-BCB molecules based on the attachment energies.

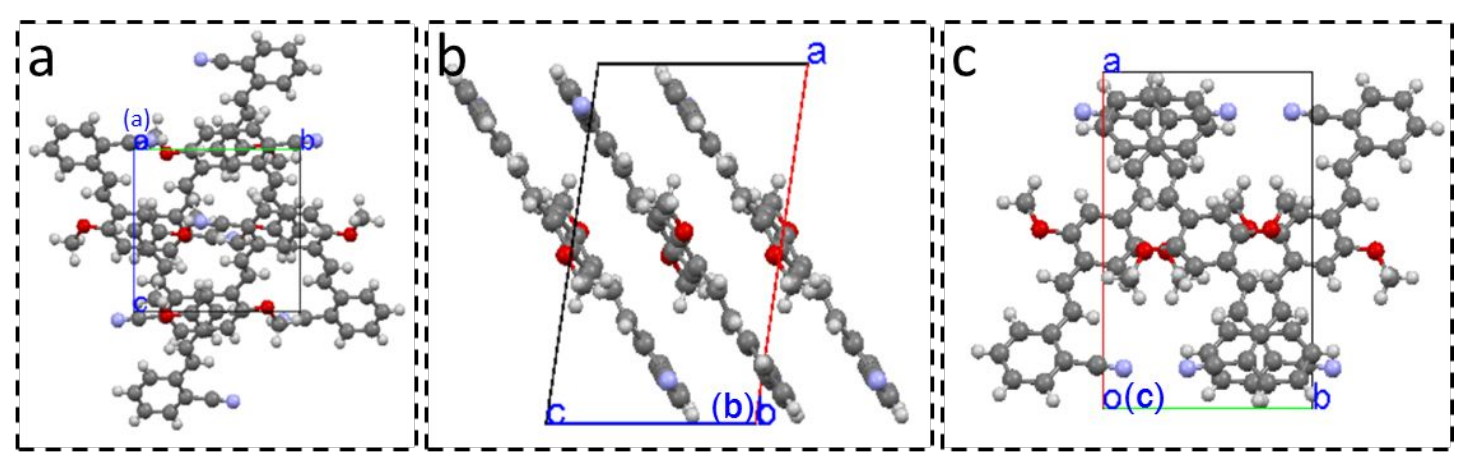

Figure S8. The unit cell structure of $m$-BCB crystals from different views along (a) $a$ axis;

(b) $b$ axis; (c) $c$ axis. 


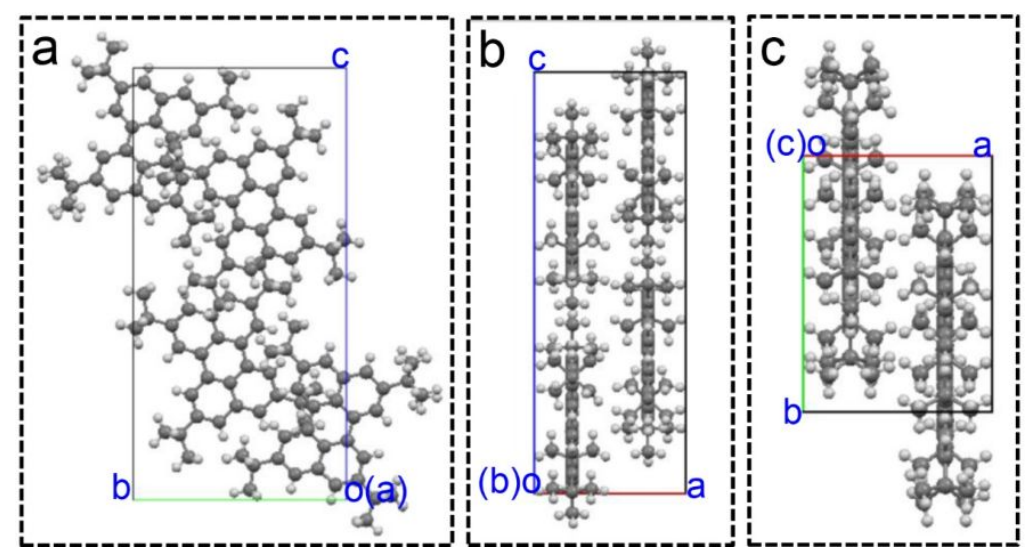

Figure S9. The unit cell structure of TBPe crystals from different views along (a) $a$ axis; (b) $b$ axis; (c) $c$ axis.
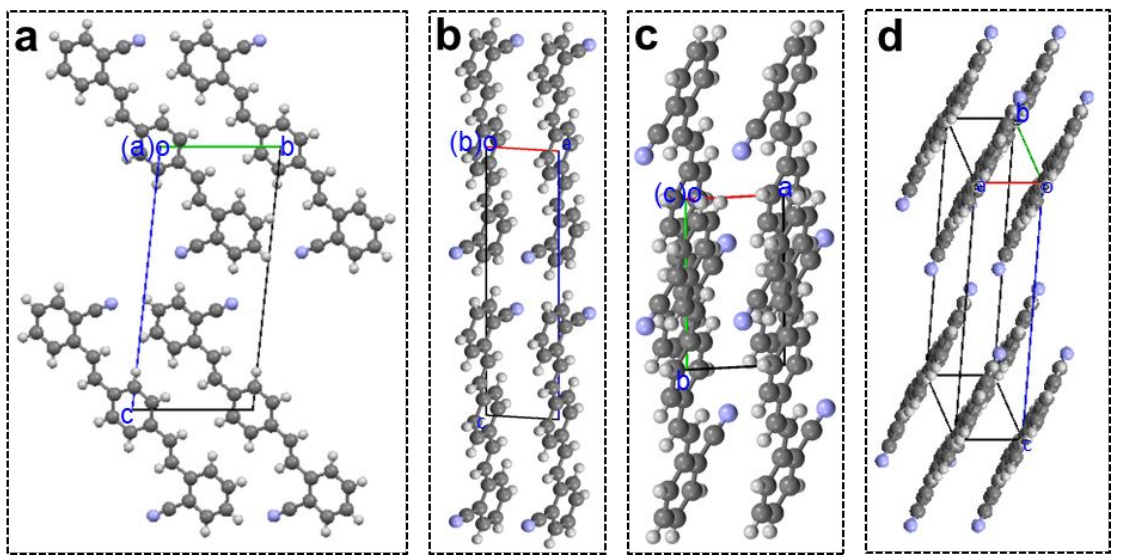

Figure S9. The unit cell structure of $o$-BCB crystals from different views along (a) $a$ axis;

(b) $b$ axis; (c) $c$ axis; (d) [011] direction. 

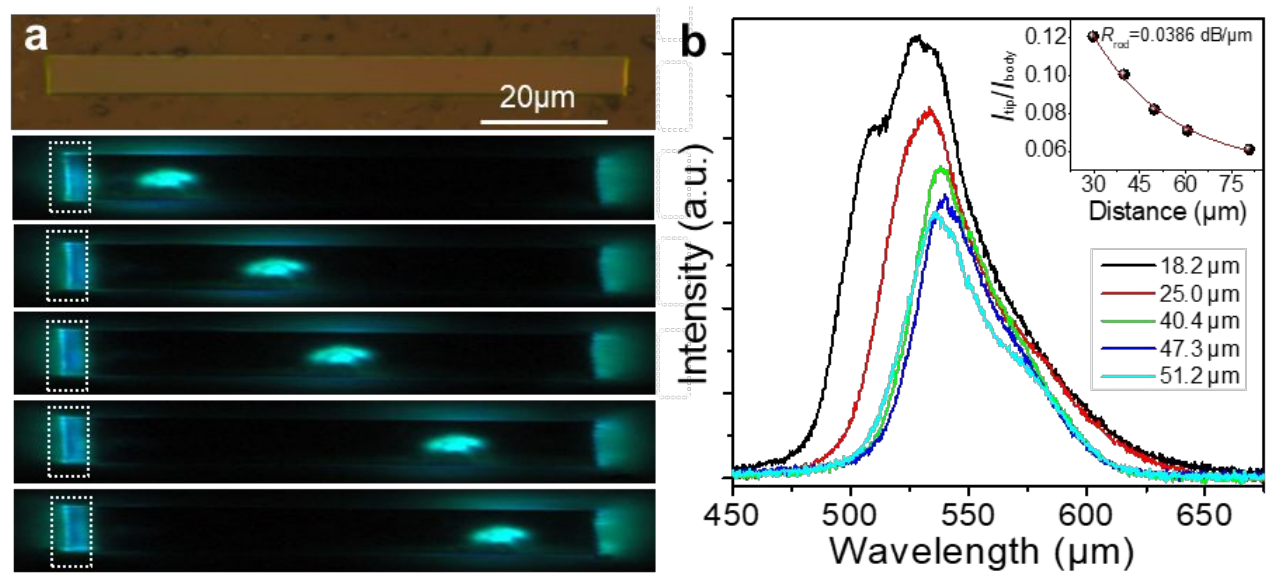

Figure S10. FM images obtained from an individual (a) $o$-BCB microwire by exciting with a laser beam $(\lambda=375 \mathrm{~nm})$ at different positions with a scale bar of $20 \mu \mathrm{m}$. (b) Corresponding spatially resolved PL spectrum in (a) with different separation distances. Inset: The ratios of the intensity $I_{\text {tip }} / I_{\text {body }}$ against the distance $d$. Curves were fitted by an exponential decay function $I_{\text {tip }} / I_{\text {body }}=\operatorname{Aexp}(-\mathrm{R} d)$. 
Table S1. Crystal data and structure refinement for $o$-BCB (CCDC No. 979756), $m$-BCB (CCDC No. 1987448), and TPBe (CCDC No. 1987449).

\begin{tabular}{|c|c|c|c|}
\hline Name & $o$-BCB & $m$-BCB & TBPe \\
\hline CCCD No. & 979756 & 1987448 & 1987449 \\
\hline Empirical formula & $\mathrm{C}_{24} \mathrm{H}_{16} \mathrm{~N}_{2}$ & $\mathrm{C}_{26} \mathrm{H}_{20} \mathrm{~N}_{2} \mathrm{O}_{2}$ & $\mathrm{C}_{36} \mathrm{H}_{44}$ \\
\hline Formula weight & 332.4 & 392.3 & 476.7 \\
\hline Temperature & $296(2) \mathrm{K}$ & $193(2) \mathrm{K}$ & $292(2) \mathrm{K}$ \\
\hline Wavelength & $0.71073 \AA$ & $1.34139 \AA$ & $0.71073 \AA$ \\
\hline Crystal system & Triclinic & Monoclinic & Orthorhombic \\
\hline Space Group & $P-1$ & $\mathrm{P} 2{ }_{1} / \mathrm{c}$ & $\mathrm{P}-1$ \\
\hline \multirow[t]{3}{*}{ Cell Lengths $(\AA ̊)$} & $a=3.9127(6)$ & $a=13.691(14)$ & $a=9.284(10)$ \\
\hline & $b=6.9657(12)$ & $b=8.940(8)$ & $b=12.650(14)$ \\
\hline & $c=15.611(2)$ & $c=8.332(9)$ & $c=26.028(3)$ \\
\hline \multirow[t]{3}{*}{ Cell Angle $\left(^{\circ}\right)$} & $\alpha=95.798(12)$ & $\alpha=90$ & $\alpha=90$ \\
\hline & $\beta=93.445(12)$ & $\beta=98.78$ & $\beta=90$ \\
\hline & $\gamma=92.199(13)$ & $\gamma=90$ & $\gamma=90$ \\
\hline Cell Volume $\left(\AA^{3}\right)$ & 422.108 & 1007.87 & 3056.84 \\
\hline Z Z & $Z: 1 Z^{\prime}: 0$ & $Z: 2 Z^{\prime}: 0$ & $Z: 2 Z^{\prime}: 0$ \\
\hline
\end{tabular}


Table S2. Attachment energies of various crystal facets $\{h k l\}$ s of $m$-BCB and TPBe crystal calculated by using the Materials Studio software package.

\begin{tabular}{|c|c|c|c|}
\hline$h k l$ & $d_{\mathrm{hkl}}(\AA)$ & Surface Area & $E_{\text {attch }}(\mathrm{kcal} / \mathrm{mol})$ \\
\hline \multicolumn{4}{|c|}{$m-\mathrm{BCB}$} \\
\hline$\left\{\begin{array}{lll}1 & 0 & 0\end{array}\right\} \mathrm{s}$ & 13.54 & 74.61 & -21.53 \\
\hline$\left\{\begin{array}{lll}0 & 1 & 0\end{array}\right\} \mathrm{s}$ & 8.94 & 112.98 & -104.98 \\
\hline$\left\{\begin{array}{lll}1 & 1 & 0\end{array}\right\} \mathrm{s}$ & 7.46 & 135.40 & -68.04 \\
\hline$\left\{\begin{array}{lll}0 & 1 & 1\end{array}\right\} \mathrm{s}$ & 6.06 & 166.77 & -72.15 \\
\hline$\{11-1\} \mathrm{s}$ & 5.79 & 174.56 & -66.69 \\
\hline \multicolumn{4}{|c|}{$o$-BCB } \\
\hline$\left\{\begin{array}{lll}0 & 0 & 1\end{array}\right\} \mathrm{s}$ & 15.50 & 27.23 & -7.50 \\
\hline$\left\{\begin{array}{ll}0 & 1-1\end{array}\right\} \mathrm{s}$ & 6.58 & 64.15 & -23.22 \\
\hline$\left\{\begin{array}{lll}0 & 1 & 1\end{array}\right\} \mathrm{s}$ & 6.09 & 69.30 & -27.81 \\
\hline$\left\{\begin{array}{lll}1 & 0 & 0\end{array}\right\} \mathrm{S}$ & 3.90 & 108.19 & -36.79 \\
\hline \multicolumn{4}{|c|}{$\mathrm{TBPe}$} \\
\hline$\left\{\begin{array}{lll}0 & 0 & 1\end{array}\right\} \mathrm{s}$ & 26.03 & 117.44 & -38.06 \\
\hline$\left\{\begin{array}{lll}0 & 1 & 0\end{array}\right\} \mathrm{s}$ & 12.65 & 241.64 & -127.15 \\
\hline$\left\{\begin{array}{ll}0 & 1-1\end{array}\right\} \mathrm{s}$ & 11.38 & 268.67 & -126.64 \\
\hline$\left\{\begin{array}{lll}1 & 0 & 0\end{array}\right\} \mathrm{s}$ & 9.28 & 329.26 & -95.50 \\
\hline$\left\{\begin{array}{lll}1 & 1 & 0\end{array}\right\} \mathrm{s}$ & 7.48 & 408.42 & -143.37 \\
\hline
\end{tabular}

